\title{
Precision Limits to STEM Imaging from Dynamical Scattering and Channeling of Sub-Angstrom Electron Probes
}

\author{
Andrew B. Yankovich and Paul M. Voyles \\ Dept. of Materials Science and Engineering, University of Wisconsin, Madison, WI 53706, USA.
}

Precision smaller than the resolution is routinely achieved in electron microscopy, with 3-5 pm being the best reported for STEM [1], compared to 1-3 pm for TEM [2]. The signal to noise ratio (SNR) introduces the fundamental precision limit in STEM and TEM images. However, before the fundamental limit is reached, practical limits are usually encountered, such as image distortions caused by instabilities of the electron probe and sample position. We have recently developed a non-rigid (NR) registration technique that accounts for all types of image distortions by allowing each pixel in each image its own deformation [3][4][5]. The NR registration and averaging of a HAADF STEM image series greatly increases the image SNR and allows for sub-pm precision, 5-7 times better than rigid registration on the same series of images. NR registering and averaging image series of $\mathrm{Pt}$ and $\mathrm{Au}$ nanocatalysts have allowed pm-scale measurements of the surface atom bond length variation that may help explain their catalytic activity. In addition, NR registration on the Pt and Au nanocatalyst data allows for standardless atom counting with the uncertainty no longer dominated by Poisson noise.

The realization of sub-pm precision in STEM imaging using NR registration leads to the question: what are the limits to precision imposed by dynamical scattering of the electron wave? Is the center of the atomic column in the image always and precisely the position of the atomic column in sample? Past studies [6] suggest that the imaged structure in some zone axis STEM images is not a perfect representation of the sample structure, calling into question the robustness and interpretability of STEM images over sample thicknesses at extreme precision.

We have used frozen phonon multislice simulations utilizing the Kirkland implementation to explore the precision limits in ABF and HAADF STEM images introduced by electron beam channeling and dynamical scattering in $\mathrm{Si}$ and $\mathrm{GaN}$ single crystals. Figure 1 shows the $\mathrm{Si}-\mathrm{Si}$ dumbbell separation distance as a function of sample thickness in (a) HAADF and (b) ABF images of Si [110]. Figure 2(a) shows the Ga position as a function of sample thickness in HAADF images of GaN [11 $\overline{2} 0$ ]. Figure 2(b) shows the Ga-N dumbbell separation distance as a function of sample thickness in ABF images of GaN [11 20 ]. For $\mathrm{Si}$, a precision limit of $\sim 2 \mathrm{pm}$ for HAADF and $\sim 14 \mathrm{pm}$ for ABF images is introduced from the electron beam channeling and dynamical scattering. For $\mathrm{GaN}$, a precision limit of $\sim 1 \mathrm{pm}$ for HAADF and $18 \mathrm{pm}$ for ABF images is introduced.

These results highlight that the position of atomic columns in HAADF are robust as a function of thickness down to $\sim 1 \mathrm{pm}$, while for ABF STEM images atomic columns could displaced from their correct position by $10-20 \mathrm{pm}$. They also point to continued need for detailed image simulations to interpret column positions as well as intensities in STEM images. That in turn requires highly accurate local determination of sample thickness, which may be possible using techniques like position averaged convergent beam electron diffraction (PACBED) [7].

For $\mathrm{Si}$, the atom motions as a function of thickness follow a breathing motion of the dumbbell for both imaging modes. Some similar motion is observed for GaN, although it is less clear. The motion can be 
explained by channeling of the electron probe and by the interference of close proximity 1s Bloch states of the crystal that varies with sample thickness [6]. Additional simulations of probe propagation and its relationship with the atomic column positions in the images will be discussed.

\section{References:}

[1] K. Kimoto et al., Ultramicroscopy 110, 778 (2010).

[2] Kisielowski et al., Phil. Mag. 86, 4575, (2006).

[3] B. Berkels et al., Microscopy and Microanalysis 18 suppl. 2, 300-301 (2012).

[4] A.B. Yankovich et al., Microscopy and Microanalysis 19 suppl. 2, 16881689 (2013).

[5] B. Berkels et al. Ultramicroscopy, in press (2014).

[6] R. Hovden et al., Phys. Rev. B. 86, 195415 (2012).

[7] J. LeBeau et al, Ultramicroscopy 110, 118 (2010).

[8] Acknowledgements: Work was funded by the Department of Energy, Basic Energy Sciences (DE-FG0208ER46547).
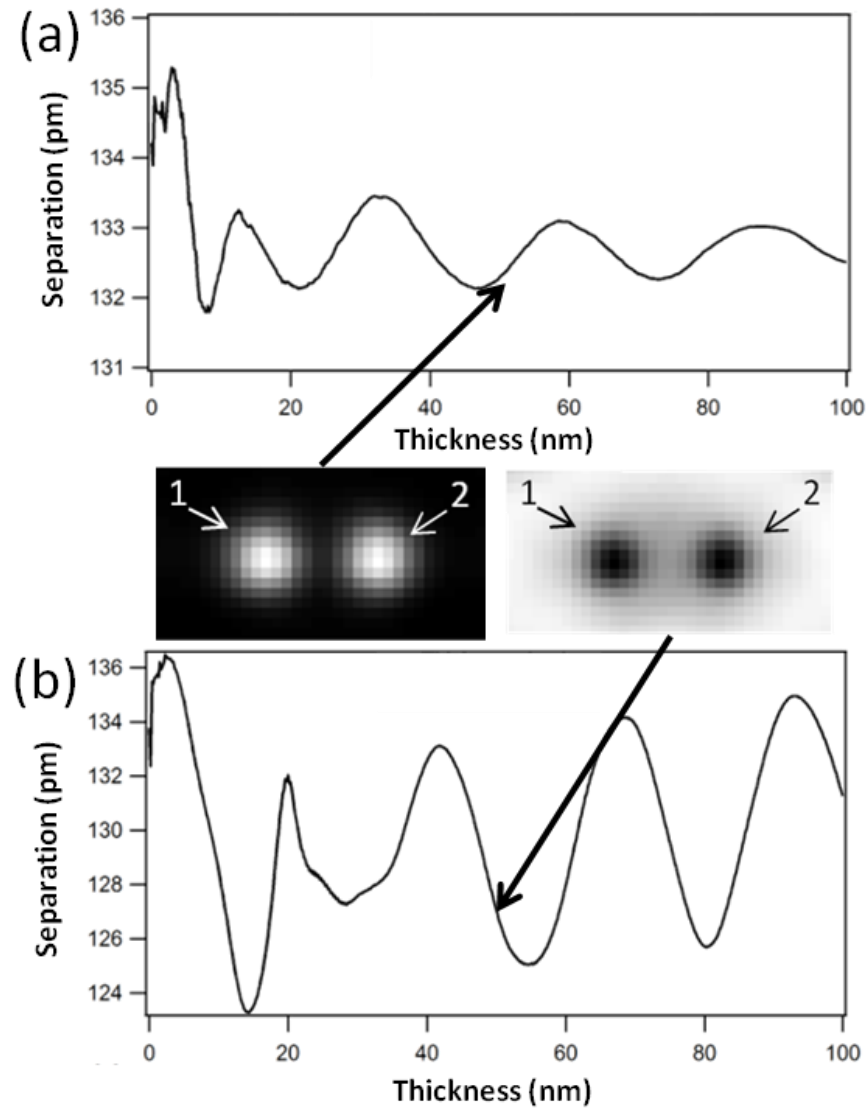

Figure 1: $\mathrm{Si}$ [110] dumbbell separation distance as a function of sample thickness for (a) HAADF and (b) ABF STEM images. The inset images are the simulated HAADF and ABF STEM images of a sample thickness of $50 \mathrm{~nm}$, as shown by the arrows that point to their location on the plots.
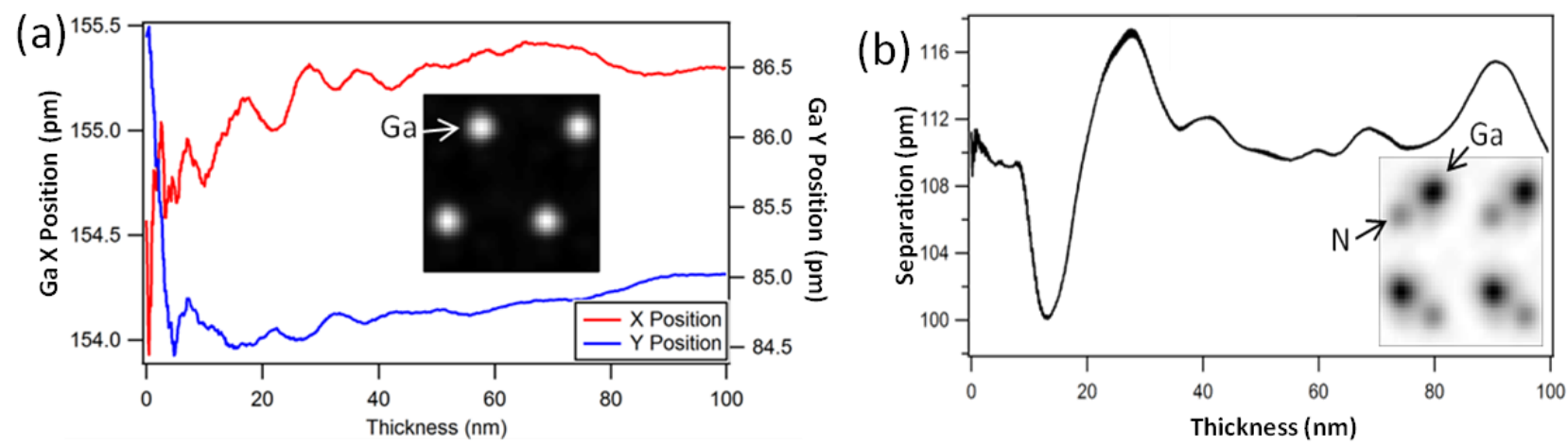

Figure 2: (a) Ga x and y position in HAADF STEM images of GaN [11 0] as a function of sample thickness. (b) GaN [ 1110$]$ dumbbell separation distance as a function of sample thickness for ABF STEM images. The insets are the HAADF and $\mathrm{ABF}$ images at a thickness of $50 \mathrm{~nm}$. 